\title{
Comparison of quality of life in patients with androgenetic alopecia and alopecia areata*
}

\author{
Muzeyyen Gonul ${ }^{1}$, Bengu Cevirgen Cemil ${ }^{1}$, Havva Hilal Ayvaz $^{1}$, Eylem Cankurtaran², Can Ergin ${ }^{1}$ \\ Mehmet Salih Gurel ${ }^{3}$
}

DOI: http:/ / dx.doi.org/10.1590/abd1806-4841.20186131

\begin{abstract}
BACKGROUND: Androgenetic alopecia is one of the most common forms of hair loss. Alopecia areata is a common autoimmune disorder which causes hair loss. It has been previously reported that both alopecia disorders can have negative effects on quality of life. However, only a few studies have compared the effects of the two disorders.

OвJестіve: The aim is to show the impact of alopecia on patients' quality of life and compare patients with androgenetic alopecia and alopecia areata.

Methods: 82 androgenetic alopecia and 56 alopecia areata patients were recruited. All patients were evaluated with the Hairdex scale and dermatology quality of life instrument in Turkish (TQL), and the scores were statistically compared according to age, sex, employment and education status, and severity of illness in the two groups. Also, female patients were statistically evaluated according to whether they wore headscarves.

RESULTS: Androgenetic alopecia patients had significantly higher total Hairdex scores in terms of emotions, functioning, and symptoms, while self-confidence was significantly higher in the alopecia areata patients. No significant differences were found in stigmatization or TQL scores between groups. The Hairdex scale and TQL scores did not show differences between the groups in terms of wearing headscarves.

STUDY LIMITATIONS: The validity and reliability of the Hairdex index have not been established in Turkey.

CONCLUSIONS: Based on the Hairdex scale, our findings revealed that androgenetic alopecia patients are more affected by their disorder than alopecia areata patients. Although androgenetic alopecia is common and neither life-threatening nor painful, it is a stressful disorder with increased need for improvement in the patient's quality of life.
\end{abstract}

Keywords: Alopecia; Alopecia areata; Female; Male; Quality of life

\section{INTRODUCTION}

Hair loss is a worldwide problem. Androgenetic alopecia (AGA) is a common hair loss disorder with a genetic predisposition that can occur in both sexes and at any age after puberty. ${ }^{1}$ AGA is biologically benign and is not a disease in the conventional sense. ${ }^{2}$
Alopecia areata (AA) is characterized by sudden onset hair loss in well-circumscribed round/oval patches without scarring. In severe cases, hair loss can involve the entire scalp (alopecia totalis) or body (alopecia universalis). ${ }^{3}$

\footnotetext{
Received 07 June 2016.

Accepted 04 June 2017.

Work conducted at the Department of Dermatology, University of Health Sciences, Dışkapı Yıldırım Beyazıt Training and Research Hospital, Ankara, Turkey. Financial support: None.

Conflict of interest: None.

Department of Dermatology, University of Health Sciences, Dışkapı Yıldırım Beyazıt Training and Research Hospital, Ankara, Turkey. Department of Psychiatry, University of Health Sciences, Dışkap1 Yıldırım Beyazıt Training and Research Hospital, Ankara, Turkey. Department of Dermatology, University of Istanbul Medeniyet, Istanbul, Turkey.
}

MAILING AdDRESS:

Bengu Cevirgen Cemil

E-mail: dbcemil@yahoo.com

(C)2018 by Anais Brasileiros de Dermatologia 
A Turkish QOL instrument (TQL)

Select the answer that best describes your situation in the last month, for each of the following questions. If the question is not related to your condition, you may simply mark the option "never = not at all". The information you give here is for research and will not be used for any other purpose. Your participation is greatly appreciated

1. Do you feel uncomfortable, frustrated, or stressed because of your skin disease?
a) Always
b) Often
c) Sometimes
d) Rarely
e) Never $=$ Not at all

2. Do you feel that your physical appearance has deteriorated due to your skin disease, and do you look in the mirror too often or avoid looking in the mirror entirely?
a) Always
b) Often
c) Sometimes
d) Rarely
e) Never $=$ Not at all

3. Does your skin disease prevent you from doing household chores and negatively affect your school and/or work life?
a) Always
b) Often
c) Sometimes
d) Rarely
e) Never $=$ Not at all

4. Do you feel uncomfortable when people in the community ask you about your skin condition, reminding you of the disease?
a) Always
b) Often
c) Sometimes
d) Rarely
e) Never $=$ Not at all

5. Because of your skin disease, do you avoid your friends or refrain from attending social situations?
a) Always
b) Often
c) Sometimes
d) Rarely
e) Never $=$ Not at all

6. Have problems from your skin disease (like bleeding, wounds, pain, itching, patches) limited your daily life?
a) Always
b) Often
c) Sometimes
d) Rarely
e) Never $=$ Not at all.

7. Do you think people stay away or avoid close contact with you (such as shaking hands or kissing) because of your skin disease? Do you avoid people for this reason?
a) Always
b) Often
c) Sometimes
d) Rarely
e) Never $=$ Not at all

8. Has your sex life been affected because of your skin disease?
a) Always
b) Often
c) Sometimes
d) Rarely
e) Never $=$ Not at all

9. Do you get desperate thinking that your skin disease will never improve or will relapse?
a) Always
b) Often
c) Sometimes
d) Rarely
e) Never $=$ Not at all

10. When treating your skin disease, have you ever thought that you are just wasting time and money?
a) Always
b) Often
c) Sometimes
d) Rarely
e) Never $=$ Not at all

11. Does your skin disease keep you from eating what you want, getting dressed, grooming, or bathing?
a) Always
b) Often
c) Sometimes
d) Rarely
e) Never $=$ Not at all 
Hair is an important component of identity and self-image. Hair loss can lead to a variety of psychological difficulties and have a negative impact on quality of life (QoL) ${ }^{2,4}$ Loss of self-confidence, lowered self-esteem, and heightened self-consciousness are common responses to hair loss, particularly for women. ${ }^{5,6}$ People with alopecia are more likely to develop depression and anxiety. ${ }^{5}$

AA and AGA patients were evaluated separately with different dermatology-specific questionnaires to assess QoL. However, these two diseases did not compare with the hair-specific QoL index. .,2,-6 $^{-1}$ The main aim of this study was to assess QoL in patients with AA or AGA according to the Hairdex and Dermatology QoL instrument in Turkish (TQL), and to compare these results between the two groups (Chart 1).

\section{METHODS}

\section{Study Design and Patients}

The protocol for this study was approved by the Research Ethics Committee of the Diskapi Yildirim Beyazit Educational and Research Hospital, and informed consent was obtained from all of the patients.

Eighty-two patients with AGA and 56 patients with AA that were seen at the dermatology outpatient clinic were included in this study. Additional inclusion criteria for the study group were age greater than 18 years, literacy, and absence of psychiatric disorders. Demographic and clinical data were collected on each patient. Age, gender, educational status, profession, duration of disease, and severity of disease were recorded. In male and female AGA patients, Hamilton Norwood scale and Ludwig scale, respectively, were used to estimate disease severity. In AA patients, disease severity was categorized as follows: focal, multifocal (one part), multifocal (more than one part), or alopecia totalis. Alopecia universalis patients were excluded.

Patients were evaluated for their QoL using the TQL and Hairdex scales. TQL consists of 11 questions, each related to a different aspect of skin disease (social, emotional, cognitive, sex life, daily activity, and symptoms) and QoL. ${ }^{7}$ Answers were scored from 0 to 4 according to the grading system. The maximum score was 44 , and higher scores indicated lower quality of life. Hairdex is an instrument developed to measure QoL in patients with disorders of the hair and scalp. The scale includes questions in five categories: a) emotions; b) functioning; c) symptoms; d) self-confidence; and e) stigmatization. Answers were graded from 0 to 4 by the subjects according to the frequency with which they occurred. Higher subscale scores for emotions, functions, symptoms, and stigmatization indicate more adverse effects on QoL, but a lower score on the self-confidence subscale suggests lower self-confidence or more adverse effect on QoL. ${ }^{8-11}$

TQL and Hairdex scores obtained in the AGA and AA groups were compared statistically. Scores were compared according to age, sex, employment status, schooling, and severity of disorder in the two groups. Also, female patients were statistically evaluated according to whether they wore headscarves.

Statistical Analysis

SPSS (Statistical Package for the Social Sciences, version
16.0) was used to analyze the data. Descriptive statistics for variables were expressed as means \pm standard deviations. The independent sample t-test, Pearson correlation coefficient, chi-square test, and ANOVA were used to analyze AGA and AA patient data. A p-value of less than 0.05 was considered statistically significant for all the analyses.

\section{RESULTS}

Of the 82 AGA patients, 52 were female (63.4\%) and 30 (36.6\%) were male. Of the AA 56 patients, there were 25 females $(44.6 \%)$ and 31 males (55.4\%). Mean ages were $30.13 \pm 11.10$ years for AGA patients and $29.34 \pm 8.13$ years for AA patients. Duration of disease was $37.68 \pm 35.77$ months and $44.30 \pm 73.41$ months for AGA and AA, respectively. There were no statistically significant differences in age, duration of disease, or schooling between AGA and AA patients. Table 1 shows the main clinical characteristics.

Patients with AGA had significantly higher total Hairdex and Hairdex subgroup scores in terms for emotions, functioning, and symptoms, while the difference in stigmatization was not statistically significant. However, self-Confidence was significantly lower in AGA patients (Table 2). No significant differences were found in TQL scores between the two groups (Table 2). In patients with AGA, no statistically significant differences were found in total Hairdex, Hairdex subgroup scores, and TQL scores according to sex, employment, or schooling (Table 3). In female patients with AGA, elevated scores of Hairdex subgroup for symptoms were seen with increasing Ludwig stages (Figure 1). Meanwhile, lower Hairdex subgroup scores for symptoms were associated with higher Hamilton stages in male patients with AGA (Figure 2).

Total Hairdex and Hairdex subgroup scores for emotions and symptoms were significantly higher in female patients with AA (Table 3). There was no statistically significant difference in Hairdex scores of AA group according to schooling. In addition, statistically higher Hairdex subgroup scores for emotions, functioning, stigmatization, and self-confidence, total Hairdex, and TQL scores were seen in AA patients with increasing disease severity. Unemployed patients with AA had higher total Hairdex and Hairdex subgroup scores for emotions, functioning, and symptoms. Meanwhile, employed patients with AA scored higher in the Hairdex subgroup for self-confidence (Table 4).

AGA and AA patients were divided into two groups based on age in years (Group 1: 18-28 and Group 2: 29+). In AGA and AA patients, there was no statistically significant difference between younger and older patients according to total Hairdex, Hairdex subgroups, or TQL.

In AGA patients, there was no statistically significant correlation between duration of disease and Hairdex or TQL scores. Meanwhile, there was a positive correlation between duration of disease and scores in terms of total Hairdex and Hairdex subgroup scores for emotions, functioning, symptoms, and stigmatization, and TQL scores in AA patients. Interestingly, the results showed a negative correlation between duration of disease and self-confidence (Table 5).

Hairdex scale and TQL scores showed no difference between the groups in female patients according to wearing headscarves. 
TABLE 1: Patients' main clinical characteristics

Number of patients

Age (mean \pm standard deviation)

Duration of disease in months (mean \pm standard deviation)

Schooling

(number of patients)

Employment status

(number of patients)

Headscarves

(number of patients)

Severity of

disease (number

of patients)

Ludwig

(n)

Hamilton

AA

\begin{tabular}{cccc}
\multicolumn{2}{c}{ AGA } & \multicolumn{2}{c}{ AA } \\
\hline Female & Male & Female & Male \\
\hline 52 & 30 & 25 & 31 \\
$33.98 \pm 11.64$ & $23.47 \pm 5.79$ & $30.48 \pm 7.95$ & $28.42 \pm 8.29$ \\
$39.15 \pm 37.65$ & $35.13 \pm 32.72$ & $61.52 \pm 70.88$ & $30.42 \pm 73.58$
\end{tabular}

\begin{tabular}{|c|c|c|c|c|}
\hline Elementary & 24 & 1 & 9 & 11 \\
\hline Secondary & 15 & 17 & 7 & 15 \\
\hline University & 13 & 12 & 9 & 5 \\
\hline Student & 9 & 15 & 8 & 5 \\
\hline Employed & 10 & 14 & 9 & 24 \\
\hline Unemployed & 33 & 1 & 8 & 2 \\
\hline With & 27 & & 10 & \\
\hline Without & 25 & & 15 & \\
\hline 1 & 2 & & & \\
\hline 2 & 38 & & & \\
\hline 3 & 12 & & & \\
\hline 1 & & 6 & & \\
\hline 2 & & 9 & & \\
\hline $2 a$ & & 1 & & \\
\hline 3 & & 5 & & \\
\hline $3 a$ & & 3 & & \\
\hline 3 vertex & & 4 & & \\
\hline $5 a$ & & 1 & & \\
\hline 6 & & 1 & & \\
\hline Focal & & & 11 & 6 \\
\hline dtifocal (one part) & & & 2 & 7 \\
\hline $\begin{array}{l}\text { Multifocal } \\
\text { ore than one part) }\end{array}$ & & & 9 & 17 \\
\hline Alopecia totalis & & & 3 & 1 \\
\hline
\end{tabular}

TABLE 2: Comparison of AGA and AA patients' Hairdex and TQL scores

\begin{tabular}{|c|c|c|c|c|c|}
\hline & \multicolumn{2}{|c|}{ AGA } & \multicolumn{2}{|r|}{ AA } & \multirow[t]{2}{*}{ p-value } \\
\hline & Mean & Standard Deviation & Mean & Standard Deviation & \\
\hline Total Hairdex & 68.43 & 31.58 & 57.02 & 27.03 & 0.025 \\
\hline Emotions & 34.45 & 20.21 & 24.42 & 16.99 & 0.002 \\
\hline Functions & 5.71 & 4.07 & 3.95 & 3.40 & 0.007 \\
\hline Symptoms & 8.85 & 6.64 & 6.67 & 5.02 & 0.031 \\
\hline Self-confidence & 13.82 & 6.81 & 17.36 & 7.28 & 0.005 \\
\hline Stigmatization & 5.60 & 6.13 & 4.65 & 5.97 & 0.372 \\
\hline TQL & 14.69 & 10.15 & 13.54 & 9.85 & 0.506 \\
\hline
\end{tabular}

\section{DISCUSSION}

Alopecia is a common dermatological condition. It is typically viewed as a benign process with mostly cosmetic consequences. However, the psychological and psychosocial impact of hair loss can be significant. Over the centuries, hair has always been consid- ered a sign of beauty and health. Scalp hair is a human characteristic that conveys aspects of identity, self-image, and ethnicity. Western culture, in particular, places a premium on physical appearance. Therefore, appearance-altering conditions such as alopecia can be psychosocially distressing. Hair loss gives rise to lower personal 

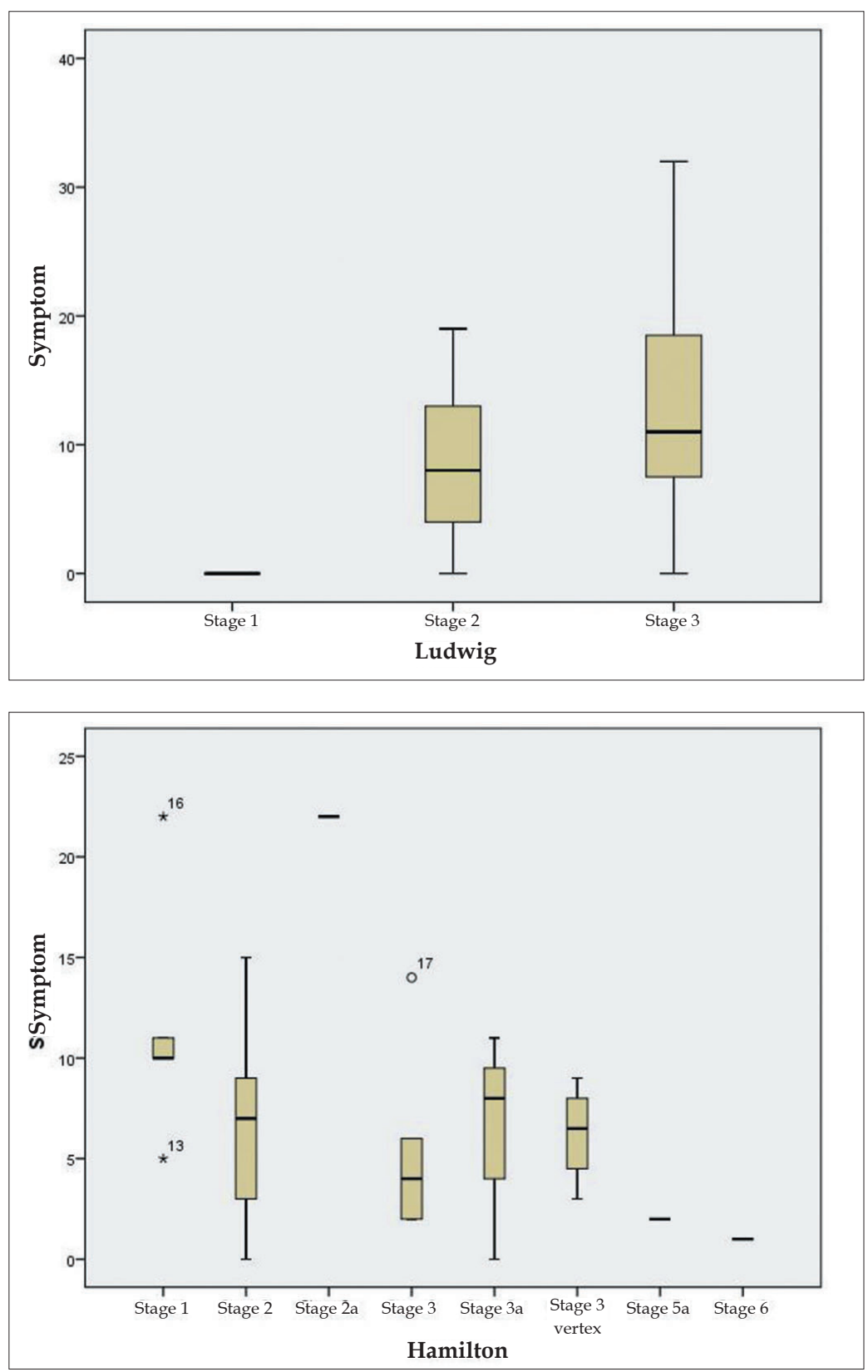

FIGURE 1: Symptom scores of female AGA patients according to Ludwig stages
Figure 2: Symptom scores of male AGA patients according to Hamilton stages attractiveness, negative self-esteem, fear of growing old, and negative repercussions on social life. ${ }^{12-14}$ Therefore, QoL assessment has become increasingly important, and many different indices that are generally assessed by self-report questionnaires are used to evaluate the effects of the disease on QoL. . $^{15,16}$

Quality of life has been evaluated previously using different scales in both AGA and AA patients, and it has been reported that these diseases impact QoL negatively. ${ }^{10,17}$

Fischer et al. developed the Hairdex score as a tool for evaluation of disease-specific quality of life in patients with hair disorders. It consists of a 48-item questionnaire with hair-specific questions, and it allows assessing the effects of hair conditions in different categories such as functioning, symptoms, emotions, self-confidence, and stigmatization..$^{8,18}$
Only a few publications have assessed QoL in alopecia patients using the Hairdex score. ${ }^{8-11}$ According to Schmidt et al., who evaluated 50 female patients with diffuse alopecia and AGA by Hairdex, diffuse hair loss had a negative impact on functioning, emotion, self-confidence, and stigmatization. ${ }^{9}$ Sawant $\mathrm{N}$ et al. evaluated QoL between two age groups of males with AGA and reported that the older age group had worse functioning, more symptoms, and higher stigmatization scores. However, emotional subscales appeared to be more affected in the younger age group. In addition, the younger age group was significantly more self-assured. ${ }^{10}$ In this study, AGA and AA patients were divided into two age groups, and there was no statistically significant difference between the two groups in total Hairdex, Hairdex subgroups, or TQL according to age. 


\begin{tabular}{|c|c|c|c|c|c|c|c|c|c|c|}
\hline & \multicolumn{5}{|c|}{ AGA } & \multicolumn{5}{|c|}{ AA } \\
\hline & \multicolumn{2}{|c|}{ Female } & \multicolumn{2}{|c|}{ Male } & \multirow{2}{*}{$\begin{array}{c}\text { p- } \\
\text { value }\end{array}$} & \multicolumn{2}{|c|}{ Female } & \multicolumn{2}{|c|}{ Male } & \multirow{2}{*}{$\begin{array}{c}\mathrm{p}- \\
\text { value }\end{array}$} \\
\hline & Mean & $\begin{array}{l}\text { Standard } \\
\text { Deviation }\end{array}$ & Mean & $\begin{array}{l}\text { Standard } \\
\text { Deviation }\end{array}$ & & Mean & $\begin{array}{l}\text { Standard } \\
\text { Deviation }\end{array}$ & Mean & $\begin{array}{l}\text { Standard } \\
\text { Deviation }\end{array}$ & \\
\hline Total Hairdex & 73.15 & 33.83 & 60.23 & 25.74 & 0.056 & 66.40 & 28.19 & 49.20 & 23.73 & 0.019 \\
\hline Emotions & 37.15 & 21.07 & 29.77 & 18.01 & 0.098 & 31.92 & 18.02 & 18.17 & 13.41 & 0.003 \\
\hline Functions & 6.31 & 4.31 & 4.67 & 3.43 & 0.062 & 3.96 & 3.70 & 3.93 & 3.18 & 0.978 \\
\hline Symptoms & 9.73 & 7.07 & 7.33 & 5.61 & 0.095 & 8.16 & 4.77 & 5.43 & 4.96 & 0.043 \\
\hline Self-confidence & 13.56 & 7.39 & 14.27 & 5.78 & 0.631 & 16.24 & 7.46 & 18.30 & 7.12 & 0.303 \\
\hline Stigmatization & 6.40 & 6.88 & 4.20 & 4.29 & 0.078 & 6.12 & 7.04 & 3.43 & 4.68 & 0.111 \\
\hline TQL & 15.48 & 11.04 & 13.28 & 8.33 & 0.314 & 16.00 & 10.90 & 11.55 & 8.59 & 0.103 \\
\hline
\end{tabular}

TABLE 4: Hairdex and TQL scores of AA patients according to employment status

\begin{tabular}{|c|c|c|c|c|c|}
\hline & \multicolumn{5}{|c|}{ AA } \\
\hline & \multicolumn{2}{|c|}{ Unemployed } & \multicolumn{2}{|c|}{ Employed } & \multirow[t]{2}{*}{ p-value } \\
\hline & Mean & Standard Deviation & Mean & Standard Deviation & \\
\hline Total Hairdex & 72.10 & 29.37 & 53.67 & 25.63 & 0.049 \\
\hline Emotions & 37.30 & 18.60 & 21.56 & 15.41 & 0.028 \\
\hline Functions & 6.20 & 3.36 & 3.44 & 3.23 & 0.034 \\
\hline Symptoms & 9.60 & 4.62 & 6.02 & 4.92 & 0.046 \\
\hline Self-confidence & 12.00 & 7.53 & 18.56 & 6.75 & 0.026 \\
\hline Stigmatization & 7.00 & 6.93 & 4.13 & 5.69 & 0.246 \\
\hline TQL & 18.80 & 9.74 & 12.39 & 9.60 & 0.081 \\
\hline
\end{tabular}

TABLE 5: Correlation of AGA and AA patients Hairdex and TQL scores and disease duration

\begin{tabular}{lcccc} 
& \multicolumn{2}{c}{ AGA } & \multicolumn{2}{c}{ AA } \\
\cline { 2 - 5 } & $\begin{array}{c}\text { Correlation } \\
\text { Coefficient }\end{array}$ & $\begin{array}{c}\text { p- } \\
\text { value }\end{array}$ & $\begin{array}{c}\text { Correlation } \\
\text { Coefficient }\end{array}$ & $\begin{array}{c}\text { p- } \\
\text { value }\end{array}$ \\
\cline { 2 - 5 } Total Hairdex & 0.11 & 0.318 & 0.44 & $\mathbf{0 . 0 0 1}$ \\
Emotions & 0.09 & 0.415 & 0.47 & $\mathbf{0 . 0 0 1}$ \\
Functions & 0.05 & 0.661 & 0.35 & $\mathbf{0 . 0 0 8}$ \\
Symptoms & 0.01 & 0.915 & 0.29 & $\mathbf{0 . 0 3 0}$ \\
Self Confidence & 0.06 & 0.586 & -0.29 & $\mathbf{0 . 0 3 3}$ \\
Stigmatization & 0.16 & 0.149 & 0.54 & $\mathbf{0 . 0 0 1}$ \\
TQL & -0.12 & 0.294 & 0.42 & $\mathbf{0 . 0 0 1}$ \\
\hline
\end{tabular}

To our knowledge, there have been only three studies comparing patient QoL with AA and AGA using different scales with varying results. ${ }^{19-21}$ Reid EE et al. evaluated QoL in female patients with AA, AGA, and telogen effluvium by Skindex-16 and reported that mean global Skindex-16 scores did not differ significantly across hair loss types. However, the Skindex-16 symptoms domain showed significant differences among hair loss types, with AA pa- tients scoring the lowest and AGA patients the highest. ${ }^{20}$ Yayl $\square$ Set al. evaluated QoL in AA and AGA patients and healthy controls using the Beck Depression Inventory, Beck Anxiety Inventory, and Skindex-29. They reported that QoL in patients with AA was severely impaired according to Skindex-29 and all three sub-units of this test (symptomatic, functional, and emotional). ${ }^{19}$ According to a study by Cartwright $\mathrm{T}$ et al. in which $48 \%$ of cases were alopecia areata, $28 \%$ alopecia universalis, 13\% alopecia totalis, and 9\% AA (the remainder were unclassified), QoL was significantly lower in patients with AGA than in all three other types, as measured by the Dermatology Life Quality Index (DLQI). ${ }^{21}$ All of these studies were performed with nonspecific scales for hair disorders. Our study evaluated QoL in AGA and AA patients using both TQL and Hairdex, both of which are specific scales for hair disorders. Moreover, the QoL of patients with AGA and AA was assessed according to age, gender, educational level, occupation, Ludwig and Hamilton grades in AGA patients, severity of illness in AA patients, duration of illness, and wearing headscarves.

The study found statistically significant differences for several parameters according to Hairdex, but the same was not true for TQL. We thus believe that the effects of hair disorders on QoL should be assessed by specific scales such as Hairdex, which would be more reliable in this case. 
In this study, specific scores for functioning, emotions, and symptoms were significantly higher in AGA patients when the groups were compared according to Hairdex subgroups. In contrast, self-confidence was significantly higher in AA patients. There was no statistically significant difference in stigmatization scores. The results show that QoL in AGA patients is more affected than originally thought, even though it is considered biologically benign.

It has been reported that women with alopecia experience specific psychosocial problems including loss of self-confidence, low self-esteem, and heightened self-consciousness. Women also report feeling upset, angry, and worried in response to their condition. ${ }^{20}$ Hair is also likely to play a more pivotal role in identity and self-image for women. In line with social stereotypes, men were more likely to express concern over interference in their work, whereas women were more concerned about the social impact of such a visible condition. Cartwright $\mathrm{T}$ et al. reported that women with alopecia showed worse illness perceptions, greater difficulty in coping, and lower QoL when compared to men. ${ }^{21}$ Our study produced similar results to the literature, and the AA group of female patients scored statistically higher in total Hairdex and Hairdex subgroups for emotions and symptoms. Female patients with AGA also had elevated Hairdex subgroup scores in terms of symptoms observed with increasing Ludwig stages. Meanwhile, lower Hairdex subgroup scores for symptoms were seen with increasing Hamilton stages in male patients with AGA. The study showed that although men were affected by AGA, women experienced more anxiety from severe AGA, which is consistent with the literature.

A previous study showed statistically significant differences in the DLQI scores, which was observed in AA patients according to severity of the disease. DLQI scores increased with the severity of AA among the various forms of severe AA and were the highest in patients with alopecia universalis. ${ }^{22}$ In our study, and consistent with the literature, significantly increased total Hairdex and Hairdex subgroup scores for emotions, functioning, stigmatization, and self-confidence and TQL scores were observed in AA patients with increasing disease severity.

To our knowledge, no studies have evaluated QoL according to employment status of patients with AGA and AA. In the current study, unemployed AA patients scored higher on total Hairdex and Hairdex subgroups for emotions, functioning, and symptoms, while no statistically significant difference was found for total Hairdex, Hairdex subgroups, or TQL according to employment status in AGA patients. However, employed patients with AA scored higher in the Hairdex subgroup for self-confidence. Unemployed patients with AA may be more preoccupied with their appearance, because they may have more idle time.

Hairdex and TQL were assessed according to patients' educational status, and no statistically significant differences were found between the AA and AGA groups, suggesting that both groups were affected similarly by alopecia, regardless of educational level.

The current study found no statistically significant correlation between duration of disease and Hairdex and TQL scores in AGA patients. However, the study showed a positive correlation between duration of disease and total Hairdex and Hairdex subgroup scores for emotions, functioning, symptoms, and stigmatization and TQL scores, and a negative correlation between duration of disease and self-confidence in AA patients. Consistent with these results, Qi $S$ et al. found that long duration was more of an impairment to QoL in AA patients. In chronic diseases, patients would tend to adapt gradually to the disease and improve their health status over the course. While continued changes in appearance made QoL worse over time in AA patients, ${ }^{23}$ AGA patients appeared to have adapted to the disease.

Previously, Erol O et al. reported that scores on physical, psychological, and overall well-being in women wearing headscarves were lower than in women not wearing scarves. ${ }^{24}$ In the present study, Hairdex and TQL did not differ between AGA and AA patients according to whether they wore headscarves. The results confirm that hair is important for women, regardless of wearing headscarves, and that hair loss affected both groups of patients similarly.

One limitation to this study is that the validity and reliability of the Hairdex index have not been confirmed in Turkey. The development of a collecting-specific questionnaire or the investigation of the validity and reliability of an existing questionnaire is highly labor-intensive and time-consuming. Although there is no validated test in Turkey for hair disorders, the current study's findings can provide a preliminary idea on quality of life in these patients.

\section{CONCLUSIONS}

In conclusion, AGA patients are affected from the disorder more than previously thought. Dermatologists should thus be aware of this distressing condition, recognizing the increasing need for improvement in patients' quality of life. Male patients can cope with the disease over time. Alopecia has an impact on QoL regardless of educational level and may affect unemployed AA patients to a higher degree. Disease severity causes more anxiety in women, independently of whether they wear headscarves. $\square$ 


\section{REFERENCES}

1. Han SH, Byun JW, Lee WS, Kang H, Kye YC, Kim KH, et al. Quality of life assessment in male patients with androgenetic alopecia: result of a prospective, multicenter study. Ann Dermatol. 2012;24:311-8.

2. Cash TF. The psychosocial consequences of androgenetic alopecia: a review of the research literature. Br J Dermatol. 1999:141:398-405

3. Olsen EA, Hordinsky MK, Price VH, Roberts JL, Shapiro J, Canfield D, et al. National Alopecia Areata Foundation: Alopecia areata investigational assessment guidelines--Part II. National Alopecia Areata Foundation. J Am Acad Dermatol. 2004;51:440-7.

4. Hunt N, McHale S. The psychological impact of alopecia. BMJ. 2005;331:951-3.

5. Williamson D, Gonzalez M, Finlay AY. The effect of hair loss on quality of life. J Eur Acad Dermatol Venereol. 2001:15:137-9.

6. Van Der Donk J, Hunfeld JA, Passchier J, Knegt-Junk KJ, Nieboer C. Quality of life and maladjustment associated with hair loss in women with androgenetic alopecia. Soc Sci Med. 1994;38:159-63.

7. Gurel MS, Yanik M, Simsek Z, Kati M, Karaman A. Quality of life instrument for Turkish people with skin diseases. Int J Dermatol. 2005;44:933-8.

8. Fischer TW, Schmidt S, Strauss B, Elsner P. Hairdex: a tool for evaluation of diseasespecific quality of life in patients with hair diseases. Hautarzt. 2001;52:219-27.

9. Schmidt S, Fischer TW, Chren MM, Strauss BM, Elsner P. Strategies of coping and quality of life in women with alopecia. Br J Dermatol. 2001;144:1038-43.

10. Sawant N, Chikhalkar S, Mehta V, Ravi M, Madke B, Khopkar U. Androgenetic Alopecia: Quality-of-life and Associated Lifestyle Patterns. Int J Trichology. 2010;2:81-5

11. Ceren E, Ekmekci TR, Koslu A. The evaluation of quality of life in women with androgenetic alopecia using Hairdex. Şeh Tip Bülteni. 2008;42:22-6.

12. Ross EK, Tan E, Shapiro J. Update on primary cicatricial alopecias. J Am Acad Dermatol. 2005;53:1-37.

13. Sampogna F, Tabolli S, Abeni D. Impact of different skin conditions on quality of life. G Ital Dermatol Venereol. 2013:148:255-61.
14. Katoulis AC, Christodoulou C, Liakou Al, Kouris A, Korkoliakou P Kaloudi E, et al. Quality of life and psychosocial impact of scarring and non-scarring alopecia in women. J Dtsch Dermatol Ges. 2015;13:137-42.

15. Ayala F, Nino M, Fabbrocini G, Panariello L, Balato N, Foti C, et al. Quality of life and contact dermatitis: a disease-specific questionnaire. Dermatitis. 2010;21:84-90.

16. Fabbrocini G, Panariello L, De Vita V, Vincenzi C, Lauro C, Nappo D, et al. Quality of life in alopecia areata: a disease-specific questionnaire. J Eur Acad Dermatol Venereol. 2013;27:e276-81.

17. Güleç AT, Tanriverdi N, Dürü C, Saray Y, Akçali C. The role of psychological factors in alopecia areata and the impact of the disease on the quality of life. Int $J$ Dermatol. 2004;43:352-6.

18. Trüeb RM. Follow-Up. In: Trüeb RM. Female Alopecia Guide to Successful Management. Berlin: Springer; 2013. p.153-7.

19. Yayli S, Tiryaki A, Dogan S, Iskender B, Bahadir S. The Role of Stress in Alopecia Areata and Comparison of Life Quality of Patients with Androgenetic Alopecia and Healthy Controls. Türkderm. 2012:46:134-7.

20. Reid EE, Haley AC, Borovicka JH, Rademaker A, West DP, Colavincenzo M, et al. Clinical severity does not reliably predict quality of life in women with alopecia areata, telogen effluvium, or androgenic alopecia. J Am Acad Dermatol. 2012;66:e97-102.

21. Cartwright T, Endean N, Porter A. IIIness perceptions, coping and quality of life in patients with alopecia. Br J Dermatol. 2009;160:1034-9.

22. Al-Mutairi N, Eldin ON. Clinical profile and impact on quality of life: seven years experience with patients of alopecia areata. Indian J Dermatol Venereol Leprol. 2011;77:489-93

23. Qi S, Xu F, Sheng Y, Yang Q. Assessing quality of life in Alopecia areata patients in China. Psychol Health Med. 2015:20:97-102.

24. Erol 0, Can G, Aydiner A. Effects of alopecia on body image and quality of life of Turkish cancer women with or without headscarf. Support Care Cancer. 2012:20:2349-56

\section{AUTHORS'CONTRIBUTIONS}

Muzeyyen Gonul

(iD) ORCID 0000-0003-1914-2175

Approval of the final version of the manuscript, Design and planning of the study, Preparation and writing of the manuscript, Collecting, analysis and interpretation of data, Effective participation in research orientation, Intellectual participation in propaedeutic and/or therapeutic conduct of studied cases, Critical review of the literature, Critical review of the manuscript

Bengu Cevirgen Cemil

Statistical analysis, Approval of the final version of the manuscript, Preparation and writing of the manuscript, Collecting, analysis and interpretation of data, Effective participation in research orientation, Critical review of the literature, Critical review of the manuscript

Havva Hilal Ayvaz
Collecting, analysis and interpretation of data, Effective participation in research ori-
entation
$\begin{aligned} & \text { Eylem Cankurtaran } \\ & \text { Design and planning of the study, Effective participation in research orientation }\end{aligned}$
$\begin{aligned} & \text { Can Ergin } \\ & \text { Intellectual participation in propaedeutic and/or therapeutic conduct of studied cases }\end{aligned}$
$\begin{aligned} & \text { Mehmet Salih Gurel } \\ & \text { Design and planning of the study }\end{aligned}$

How to cite this article: Gonul M, Cemil BC, Ayvaz HH, Cankurtaran E, Ergin C, Gurel MS. Comparison of quality of life in patients with androgenetic alopecia and alopecia areata. An Bras Dermatol. 2018;93(5):651-8. 